\title{
OUR EXPERIENCE OF UPPER-LIMB TRANSFERS IN CASES OF TETRAPLEGIA
}

\author{
By M. Maury, M.D., M. Guillaumat, M.D. and N. François, M.D. \\ Centre de Re-education Motrice, Fontainebleau
}

IMPAIRMENT of motor function is a greater handicap in the upper limbs than in the lower. In tetraplegics the two are usually combined. Here one is more than ever divided between the wish to provide concrete aid and the anxiety not to create further disabilities. The various types of self-help aids have the advantage of not involving this risk, but the disadvantage of sometimes being very clumsy is not always acceptable to the patient, who would often rather function without external aids. For this reason, and also because any gain in independence is precious, the authors think that reconstructive surgery deserves a place in the treatment of tetraplegics, within the limited indications that shall be attempted to define. Several authors have already defended this point of view: Lipscomb, Nickel and Garrett, Freehafer, McDowell.

\section{OVERALL RESULTS}

TABLE I

\begin{tabular}{|c|c|c|c|c|}
\hline & $\begin{array}{c}\text { Much } \\
\text { improved }\end{array}$ & $\begin{array}{c}\text { Some } \\
\text { improvement }\end{array}$ & Failures & Total \\
\hline $\begin{array}{l}\text { Number of cases: } \\
\text { Ist group } \\
\text { 2nd group }\end{array}$ & $\begin{array}{l}2 \text { (2) } \\
8 \text { (I0) }\end{array}$ & $\begin{array}{ll}\text { I } & (\mathrm{I}) \\
2 & (2)\end{array}$ & $\begin{array}{ll}0 & (0) \\
4 & (5)\end{array}$ & $\begin{array}{r}3 \text { (3) } \\
14 \text { (17) }\end{array}$ \\
\hline Total & IO (I2) & 3 (3) & $4 \quad(5)$ & I7 (20) \\
\hline
\end{tabular}

Key: The figures without brackets represent the number of patients, those in brackets the number of limbs. In all, out of 17 patients (20 upper limbs) Io are considerably improved (12 upper limbs), three show some improvement (three upper limbs) and four are unchanged (five upper limbs).

In a previous article published in I968, one of our team, together with $\mathrm{P}$. Masse, Y. Bidart and B. Gravil recorded operations of this kind on nine out of Ioo tetraplegics. Today the percentage is roughly the same, since out of I76 tetraplegics treated over 20 years, 17 have undergone transfers in the upper limbs. In each of the 17 cases the lesion was considered to have stabilised and of course the possibilities of rehabilitation exhausted. Surgery was carried out after an average of 22 months from the onset of tetraplegia, the earliest being at the end of I I months and the latest after 39 months.

Among the 17 tetraplegics-representing $20^{\circ}$ upper limbs, since there are three bilateral cases-two groups can be distinguished:

I. A group of three patients who could walk normally and had no further 
sequelae of their spinal cord lesion other than a pyramidal sub-lesional syndrome and a limited lesional syndrome manifested in a unilateral and not extensive lower motor neuron impairment. Two had excellent results after transfer, one of the palmaris brevis into the abductor pollicis brevis and the extensor pollicis longus, the other of the pectoralis minor into the biceps together with an epitrochlear muscles transposition. The third, with the extensor digitorum communis and extensor carpi ulnaris in the right side, the only muscles still at 0 , underwent a transfer of the first radial into the extensor communis. This muscle only raised 0 to two, progress and function being hampered by intense spasticity of the finger flexors. Hand functioning was only slightly improved.

2. The second group, of I4 patients, included the more severe lesions, and in most cases complete, with an extensive bilateral lesional syndrome and muscle function in the upper limbs notably reduced. For all these patients it was particularly important not to add to the motor disability. in detail.

For this second group, containing the 'real tetraplegics', the results were studied

\section{ANALYSIS OF RESULTS FOR GROUP 2}

Table II sums up the 34 transfers made in this group, 27 of them involving the pronator teres ( 13 cases) or the brachioradialis ( 14 cases). The frequent choice of these two muscles is explained by their relatively high position in the segmental scale, and also by the fact that they keep their original function, at least in part, even after transfer. It can be noted immediately that the flexor digitorum profundus was re-animated I 4 times; 13 times by the pronator teres (nine successes, four failures), one by the brachioradialis (one success). Out of the 13 re-animated by the pronator teres, eight were associated with the re-animation of the flexor pollicis longus by the brachioradialis, six were successful, one slightly improved and one a failure.

Pronator teres transfered into flexor digitorum profundus + brachioradialis into flexor pollicis longus (eight cases). The only failure (observation no. 7) has two possible causes: the two transferred muscles were not intact: the pronator teres was originally evaluated at o then at three, a year later the brachioradialis at one then at four at the time of surgery. Conversely the finger flexors were spastic with one extensor digitorum communis at 0 . And in fact painful postoperative spasms occurred and then a flexor contracture. The final result was a very slight improvement of the pinch from the tenodesis effect, but very much less than was hoped.

The semi-success (observation no. 6) seemed to be due to the gradual giving of Zancoli's capsuloraphy, designed to limit hyperextension in the first phalanx of the fingers (the extensor digitorum communis was at four), certainly it is sufficient to explain why the result is not functionally better, although analytically the re-animated muscles are assessed at three + and four.

In all the successful cases, except one, the extensor digitorum communis was at three or four. In the one case where it was $O$ (observation $I$ and $I b$ ) the hands were totally flaccid. The result was good because the patient has been enabled to write, raise a glass to his mouth, shave, take off the device used for eating and dress from the waist up. Improvement in the six successful cases has always related to a variety of activities essential to daily living, several patients even managed 
PAPERS READ AT THE ANNUAL SCIENTIFIC MEETING, I973

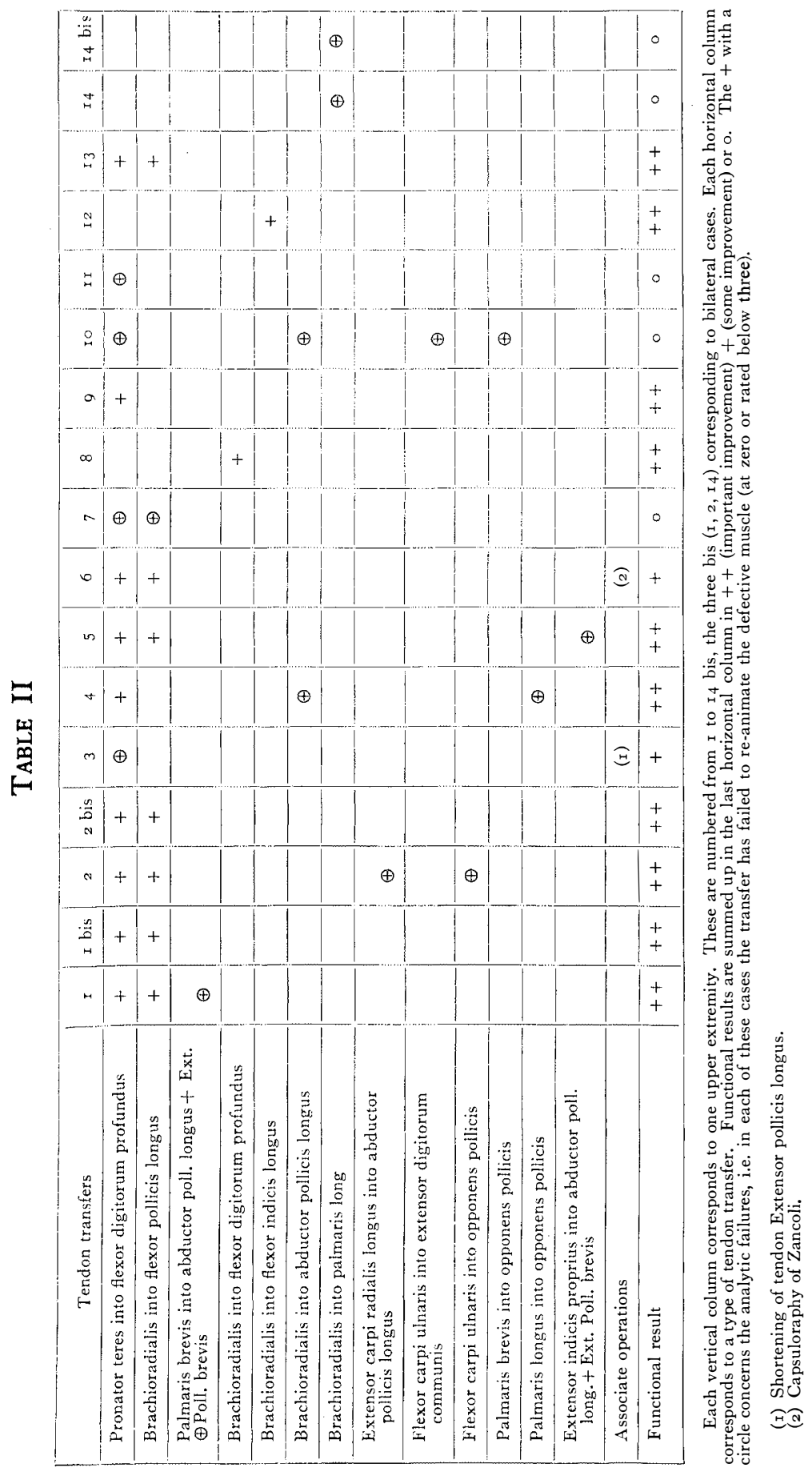

I $1 / 3-S$ 
to pull themselves up with the monkey bar to transfer from bed to wheelchair and vice versa, using the re-animated and slightly contracted flexors.

Would the results have been as good with pronator teres transfer alone? This is not known. However, it was noticed that in all cases where the brachioradialis transfer into the flexor pollicis longus was successful (seven out of eight), all the patients recovered some thumb-index pinch, not pad against pad but indexpad against the dorsal surface of the second phalanx. (The extensor pollicis longus was $O$ in all cases except one where it was assessed at three.) The disadvantage is the risk of permanent flexion of the inter-phalangeal joint and the awkwardness in certain movements that results. Even if there is no stiffness in the joint, flexion of the second phalanx during wrist extension can, for example, make it awkward to grip the hand rim of the wheels.

Pronator teres transferred into flexor digitorum profundus (five cases). In two out of five cases (observation no. 4 and 9) where the pronator teres was transferred into the flexor profundus without re-animation of the flexor pollicis longus, the result was good. For example, for the patient treated in observation no. 4, one can list the following improvements due to transfer: he eats without a self-help aid, pours a drink, writes with no special device, pulls himself up with the monkey bar, brushes his teeth, can put on his socks with a simple gadget, put on and lace up his shoes, wind up his watch, strike a match, pick up an object from the floor with his right hand and make his wheelchair go faster.

In the third case (observation 3) partial failure was due to the fact that the pronator teres was not intact, being originally assessed at $o$ then becoming spastic. It was in fact necessary to operate again, as the suture gave after two months when, the fingers were suddenly and sharply stretched during dressing. The improvement obtained was, above all, the result of shortening the long tendon extensor at the same time preventing thumb flexion-adduction being caused inside the hand and, also the result of the improved active supination obtained by reduced action of the transferred pronator teres.

The last two cases were complete failures. One (observation no. Io) experienced a difficult post-operative period with redness and painful spasms in the whole forearm for several weeks and it took a long time to recover his previous independence. A secondary tenotomy of the too-retracted flexor IV was necessary. There is no lack of reasons to explain the failure: spastic finger flexors with one extensor digitorum communis at 0 ; simultaneous re-animation of flexors and extensors. It was certainly a surgical experience that should not be repeated.

The reasons for the last failure (observation II) were more obscure. The flexors were at one and flaccid; the extensor digitorum communis assessed at three, when the post-operative position was taken off the transfer was functional; the flexors were assessed at $2+$, but they then reverted to one. It is possible that the suture did not hold.

Brachioradialis transferred into flexor digitorum profundus (one case). There was one case only of a tetraplegic with complete 0 on the right hand, radials at three, no pronator, a good brachioradialis. The first phalanx of the fingers was in extension, with no joint limitation, which led the authors to suppose that the lesion of the extensors was central (it is not always possible to know this, clinically). There was no sign of spasticity in the flexors. A myoelectrical orthesis 
had been prescribed. With the flexors assessed at $3+$ after transfer, pad-to-palm prehension became possible and effective. He no longer needed any artificial aid to eat or clean his teeth, could drink without help, hold a razor, cigarette-holder, unscrew and replace the cap of a tube of toothpaste, write with an elastic device. The myoelectrical orthesis was no longer necessary. A technical remark can be made here: the advantage of detaching the upper insertion of the brachioradialis as far up as possible, so as to completely separate it from the inter-muscle walls of the forearm, thus considerably increasing its functional length.

Brachioradialis transferred into flexor indicis profundus (one case). In this observation (no. I2) the extensor digitorum communis was at 0 while the extensor indicis proprius, with the help of spasticity, reached strength three. The finger flexors were also at $o$, but contracture caused the fingers to close in wrist extension, only the second remaining extended. Due to the transfer, the flexor indicis longus raised from 0 to four, and an excellent thumb-index pinch was achieved. It may be noted that elbow flexion in semi-pronation brought about some index flexion, which was not a particular problem.

Brachioradialis transferred into palmaris longus (two cases). This transfer failed twice in the same patient (observations I4 and I4b). It was attempted in order to bring about an increase in independence, by allowing the use of the wrist flexors to pull on the monkey bar. No explanation was found for these failures.

Other Transfers. As indicated in Table II the brachioradialis was transferred into the abductor pollicis longus twice; the extensor carpi radialis longus into the abductor pollicis longus once; the flexor carpi ulnaris into the extensor digitorum communis once and into the opponens once; the palmaris brevis into the opponens; the palmaris longus into the opponens; the extensor indicis proprius into the abductor pollicis longus and the extensor pollicis brevis. These different surgical procedures were all associated with at least one flexor digitorum profundus re-animation by the pronator teres and failed, i.e. the transfer did not function. This does not mean that there was no functional improvement. An explanation for these failures is the simultaneous re-animation of muscles which, if not completely antagonistic, have actions at least sufficiently opposing to make post-operative postures difficult to apply (abductor pollicis longus and opponens in observations 2, 4 and I0; flexor digitorum profundus and extensor indicis proprius in observation 5).

\section{FACTORS INFLUENCING THE RESULTS}

I. The transferred muscle must be above the cord lesion and its rating not less than four. When a muscle was initially assessed as nil or weak, prudence and caution is essential. In case of doubt an electromyogram determines whether the muscle in question is the seat of a peripheral impairment or not.

2. The segmentary balance must be carefully evaluated before surgery by studying the muscle testing, joint amplitudes, neurological state and, in particular signs of spasticity. Stiffness can be useful or harmful, for instance, to re-animate the flexor digitorum profundus some limitation of the metacarpo-phalangeal joints is useful, while their stiffness in hyperextension would be harmful. 
In the last four fingers re-animation of the flexor digitorum communis is particularly indicated when apart from the flexors at 0 , there are extensors of at least three (it must be remembered that the motor nuclei of the finger extensors are higher than those of the flexors, which are in turn, higher than those of the interossei-lumbricales). A certain amount of spasticity in the extensor communis may be useful. When it is 0 and flaccid, re-animation of the flexor digitorum profundus should only be attempted if there is no sign of spasticity in the flexors. Otherwise there is a risk of the fingers becoming contracted in the grasp position, or at least losing their automatic opening response associated with wrist flexion.

$\mathrm{Re}$-animation of the flexor pollicis longus (where there is a long extensor at 0 and a supple inter-phalangeal joint) always produces flexion in the inter-phalangeal joint. We are not convinced that the pinch between index pad and dorsal surface of the second phalanx is enough to justify the transfer, which is indicated, in the author's opinion, when there is a long extensor of three at least. Inter-phalangeal arthrodesis is certainly not indicated wrist extension with the re-animated flexor pollicis longus would give flexion in the first phalanx and thumb adduction. But, this adduction with permanent extension of the second phalanx would create difficulties for the closure of the hand.

This segmental balance, so important for transfers, is very much affected by the initial quality of care given to the hand commencing in the first few days of the tetraplegia. Freehafer (1969) argued this very strongly and with good reason.

3. Post-operative positions are very important in achieving the best functional results. They are governed by very simple principles: a joint tends to stiffen in the position in which it is immobilised, a position is only effective if it is maintained for the major part of 24 hours, lengthening a muscle makes it weaker, while shortening it tends to reinforce it. This is why, if the extensors are spastic, or the metacarpo-phalangeal joints are stiffened in the position of extension, it is advisable to set the re-animated flexors in the position of maximum shortening. Conversely, when attempting to re-animate the flexors with the extensors 0 and flaccid, it would seem preferable to set a position which keeps the metacarpo-phalangeal joint reasonably extended and the flexors not too short.

The position in which the patient is immobilised following surgery is discontinued after about $2 \mathrm{I}$ days, just long enough to allow progressive muscle exercises, and kept up for at least another two weeks. The joints should have their positions modified as necessary, depending on whether, and where, stiffness develops in the joints. After re-animation of the flexor digitorum profundus, one has to accept a certain amount of inter-phalangeal joint flexion due to the absence of interossei-lumbricales.

4. It seems to be preferable not to re-animate muscles which have an antagonistic or nearly antagonistic effect, if only for the good management of the postoperative position.

5. It is necessary that the patient be cooperative and desirous of improvement.

\section{CONCLUSIONS}

When segmental innervation of the upper limb muscles is considered, it is not difficult to appreciate that it may sometimes be possible to re-animate such important muscles as the finger flexors by muscles which have their nuclei situated higher up. Our experience leads us to think that the pronator teres, if normal, 
can be chosen first, and the brachioradialis can in some cases be used for the thumb at the same time. When the pronator teres is not intact, the brachioradialis, if normal, is capable of re-animating the flexor digitorum profundus.

Out of the 23 transfers of the pronator teres or the brachioradialis, or both into the finger flexors, I8 were successful.

The various other transfers performed were failures, the reasons of which are known.

This is excluding a group of three patients for whom the sequelae of the tetraplegia were relatively minimal and who underwent classical transfers for an isolated and peripheral-type impairment in the upper limbs.

None of the patients operated on sustained any lasting functional loss.

We have no experience of the brachioradialis transfer into the radials mentioned by Freehafer and Mast (1967) and Dowell (I97I). But it seems entirely logical: the radials have great functional importance, the brachioradialis keeps its function as forearm flexor where it is considerably helped by two other muscles, and the transfer can, with advantage, replace the myoelectrical orthesis adapted for such cases. So it is intended to perform this transfer if the authors are presented with radial muscles which are nil or weak with an intact brachioradialis.

Before any transfer is contemplated it is imperative to weigh up the indications most carefully (one consultation is not usually enough) between the orthopaedic surgeon, the paraplegist, the patient, his physiotherapist and his ergotherapist. The most important factors to be taken into account are: the normality of the transfer muscle, the balance of strengths and joint amplitudes in the segment of the limb in question, and the neurological state of the hand. Post-operative care is extremely important.

\section{REFERENCES}

FREeHAFER, A. A. \& MAST, W. A. (1967). Transfer of the brachioradialis to improve wrist extension in high spinal cord injury. Fournal of Bone and foint Surgery, 49A, 648.

Freehafer, A. A. (1969). Care of the hand in cervical spinal cord injuries. Paraplegia, 7-2, I I 8.

Garrett, A. L., Perry, J. \& Nickel, V. L. (I964). Traumatic quadriplegia. F.A.M.A. $187,7$.

GolDNER, J. L. (1955). Reconstructive surgery of the hand in cérebral palsy and spastic paralysis resulting from injury to the spinal cord. Fournal of bone and foint Surgery, 37A, II4I.

Lipscomb, P. R., Elkins, E. C. \& Henderson, E. D. (I958). Tendon transfers to restore function of hands in tetraplegia, especially after fracture dislocation of the sixth cervical vertebra on the seventh. Fournal of Bone and foint Surgery, 40A, I07I.

Masse, P., MAURY, M., BidarT, Y. \& GRavil, B. (I968). A propos des transplantations tendineuses pour la réanimation de la main du tétraplégique. Annales Médecine Physique, XI, 4, 35I.

Maury, M., Masse, P., Gravil, B. \& Bidart, Y. (I968). Remarques sur le membre supérieur du tétraplégique et ses possibilités fonctionnelles. Annales Médecine Physique, XI, 4, 343.

Nickel, V. L., Perry, J. \& Garrett, A. L. (1963). Development of useful function in the severely paralyzed hand. Fournal of Bone and foint Surgery, 45A, 9333.

Pulvertaft, E. (1965). Problems of flexor tendon surgery of the hand. Fournal of Bone and foint Surgery, 47, I, 123. 